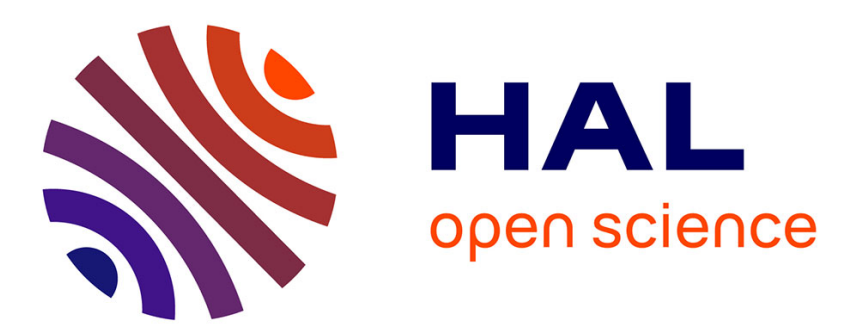

\title{
Du plomb au pixel. Transcrire les légendes des monnaies du haut Moyen Âge
}

Florence Codine-Trécourt, Guillaume Sarah

\section{To cite this version:}

Florence Codine-Trécourt, Guillaume Sarah. Du plomb au pixel. Transcrire les légendes des monnaies du haut Moyen Âge. Revue Numismatique, 2012, 168, pp.261-277. hal-01773272

HAL Id: hal-01773272

https://hal.science/hal-01773272

Submitted on 19 Dec 2018

HAL is a multi-disciplinary open access archive for the deposit and dissemination of scientific research documents, whether they are published or not. The documents may come from teaching and research institutions in France or abroad, or from public or private research centers.
L'archive ouverte pluridisciplinaire HAL, est destinée au dépôt et à la diffusion de documents scientifiques de niveau recherche, publiés ou non, émanant des établissements d'enseignement et de recherche français ou étrangers, des laboratoires publics ou privés. 


\section{REVUE NUMISMATIQUE}

Dirigée par

C. Morrisson, M. Amandry,

M. Bompaire, 0. Picard
Secrétaires de la rédaction

Fr. Duyrat, A. Hostein,

J. Jambu

2012

(169 volume) 


\title{
COMITÉ DE PUBLICATION
}

\section{DIRECTEURS}

\author{
$\mathrm{M}^{\mathrm{me}}$ Cécile Morrisson, MM. Michel Amandry, \\ Marc Bompaire, Olivier Picard
}

SECRÉTAIRES DE LA RÉDACTION

\author{
Articles \\ $\mathrm{M}^{\mathrm{me}}$ Frédérique Duyrat (frederique.duyrat@bnf.fr) \\ M. Jérôme Jambu \\ Comptes rendus \\ M. Antony Hostein (hosteinantony@yahoo.fr)
}

COMITÉ DE LECTURE

\begin{abstract}
Michael Alram, Jean Andreau, Philip Attwood, Gérard Aubin, François Baratte, Patrice Baubeau, Cécile Bresc, François de Callataÿ, Jean-Pierre Callu, Yves Coativy, Michel Christol, Michel Dhénin, Sylviane Estiot, Stefan Heidemann, Jérôme Jambu, Xavier Loriot, Marie-Christine Marcellesi, Jens Christian Moesgaard, Sylvia Nieto-Pelletier, Michel Pastoureau, Séléné Psoma, Andrea Saccocci, Thierry Sarmant, François Thierry, Lucia Travaini, Benedikt Zäch.
\end{abstract}

La Revue numismatique paraît annuellement. Elle est la propriété de la Société française de numismatique qui en est l'éditeur et en assure le service à tous ses membres à jour de cotisation pour l'année concernée, lors de sa parution. La cotisation a été fixée pour 2012 à $50 €$ et $55 €$ pour les membres résidant à l'étranger.

\author{
Société française de numismatique \\ 58, rue de Richelieu F-75002 Paris \\ http://www.sfnum.asso.fr \\ La Revue numismatique est également diffusée par \\ la Société d'édition «Les Belles Lettres» \\ 95 Boulevard Raspail, F-75006 Paris \\ Tél. : 01443984 20, Fax : 0145449288.
}

Les abonnements sont payables à la Société d'édition «Les Belles Lettres » Compte chèque postaux Paris $33657 \mathrm{P}$.

Le champ couvert par la Revue numismatique comprend la numismatique et l'histoire monétaire et s'étend à l'archéologie, l'histoire économique, l'histoire de l'art ainsi qu'à l'épigraphie, la sigillographie ou la glyptique dans leurs rapports avec l'étude des monnaies, médailles et documents monétiformes.

La Revue recherche des études de haut niveau et de première main, publication de documents nouveaux ou nouvelle interprétation de documents connus. Les articles sont retenus en fonction de leur qualité scientifique et de l'intérêt du document présenté. Les rubriques de la Revue sont indicatives et correspondent aux divisions historiques traditionnelles : numismatique celtique, grecque, romaine, byzantine, médiévale, moderne et contemporaine, orientale, médailles et jetons, histoire de la numismatique et des collections. Des notes synthétiques faisant le point sur une question ou un débat ont leur place dans les Miscellanea (la Société française de numismatique préférant réserver la publication des articles brefs au Bulletin de la Société française de numismatique).

Les langues admises sont, outre le français, l'allemand, l'anglais, l'espagnol et l'italien.

Les manuscrits complets et conformes aux instructions aux auteurs doivent être remis au secrétariat le $1^{\mathrm{er}}$ juillet de l'année qui précède la parution. Après avoir été confiés à plusieurs rapporteurs et examinés par le comité de lecture, ils sont définitivement retenus lorsque le conseil de gestion de la Revue numismatique se réunit, en janvier, pour adopter le budget de la Revue qui paraît dans l'année.

La Revue ne rend compte que des ouvrages qui sont adressés au secrétariat avec la mention «Revue numismatique». Les ouvrages sont remis à des spécialistes proposés par les directeurs au Comité de lecture. La publication rapide dans le bulletin bibliographique ne doit pas nuire au caractère informatif et critique des comptes rendus et il est possible de rendre compte simultanément et synthétiquement de plusieurs ouvrages.

La Revue numismatique se réserve le droit de refuser toute publicité sans avoir à fournir de motif à sa décision. 


\title{
Du plomb au pixel. Transcrire les légendes des monnaies du haut Moyen Âge
}

\begin{abstract}
Résumé - La publication de travaux numismatiques impose la transcription des légendes monétaires, avec plus ou moins de précision selon le but poursuivi. Un travail important a été effectué par les numismates du XIX ${ }^{\mathrm{e}}$ siècle dans ce domaine, conduisant à la réalisation de quelques jeux de caractères en plomb, désormais obsolètes face aux avancées du traitement de texte et de l'imprimerie. Ce n'est que récemment que les numismates se sont de nouveau intéressés à la question, d'autant que les nouvelles possibilités offertes par les polices de caractères numériques ouvrent des perspectives intéressantes pour la numismatique du haut Moyen Âge. La lecture des légendes, les identifications, peut-être même la chronologie et la localisation des lieux de frappe et d'émission pourraient en bénéficier, à condition de faire reposer la création d'une future police sur une étude épigraphique préalable approfondie.
\end{abstract}

Mots clés - Inscriptions, inscriptions monétaires, épigraphie, polices numériques, haut Moyen Âge.

Summary - Publishing numismatic research requires that coin inscriptions be transcribed, with more or less precision depending on the purpose of the study. 19th-century numismatists tackled the subject with some success and developed fonts which are now obsolete given the advances of typesetting. Interest in this field of research has only recently re-emerged, stimulated by the new possibilities offered by contemporary digital fonts; this is particularly true for the early Middle Ages. Interpretation of the inscriptions, the identification of places and people, and even chronology could benefit from such fonts, so long as their creation rests on a detailed preliminary epigraphic study.

Keywords - Inscriptions, coin inscriptions, epigraphy, typography, digital fonts, early Middle Ages.

\section{Introduction}

La transcription des légendes revêt une importance capitale pour l'étude des monnaies. Dans le cas du haut Moyen Âge, les grands catalogues et recueils du $\mathrm{XIX}^{\mathrm{e}}$ siècle, comme ceux de Maurice Prou et d'Auguste de Belfort, avaient pour ce faire nécessité la fonte de plombs spécifiques. Avec l'avancée des techniques d'édition, faute d'un équivalent numérique à ces fontes, les rééditions ne peuvent se faire qu'en fac-similé, procédé coûteux et qui ne permet aucune correction.

* Département des Monnaies, Médailles et Antiques, Bibliothèque nationale de France, 5 rue Vivienne, 75002 Paris. Courriel : florence.codine@bnf.fr.

** IRAMAT - Centre Ernest Babelon (CNRS), Université d'Orléans, 3D rue de la Férollerie, 45071 Orléans Cedex 2, France. Courriel: sarah@cnrs-orleans.fr. 
Le développement de la micro-informatique puis d'internet ont cependant favorisé l'éclosion de quantité de systèmes de caractères particuliers créés pour les besoins de chaque étude. Certains éditeurs et revues de numismatique se sont efforcés d'organiser et de canaliser ce foisonnement pour l'ensemble de l'épigraphie monétaire du Moyen Âge, mais sauf cas particuliers ${ }^{1}$, ces images de caractères ne sont pas indexables et interrogeables pour une recherche ultérieure. C'est en revanche envisageable avec les véritables polices numérique, de type compatible Unicode ${ }^{2}$ notamment, qui offrent également des possibilités plus grandes du point de vue de l'édition.

Au moment de développer des bases de données de recherche, individuelles ou collectives, dans le domaine du monnayage mérovingien et carolingien, il nous paraît utile de réfléchir aux perspectives ouvertes par le développement d'une police de type Unicode permettant de relier et croiser diverses bases de données (liées éventuellement à des SIG) pour faciliter l'identification et la lecture de monnaies, mais aussi pour faire des observations d'ordre chronologique et géographique sur les particularités graphiques des monnaies. Il serait aussi envisageable de croiser ces informations avec des documents écrits sur d'autres supports de la même période.

La question de l'édition des inscriptions monétaires n'est donc pas la seule qui doive être prise en considération, car les enjeux sont d'abord d'ordre scientifique. La possibilité de mener de nouvelles interrogations à partir des inscriptions monétaires (ou non monétaires) permettrait d'apporter une série de données renouvelées sinon nouvelles pour aborder l'étude des monnaies mérovingiennes et carolingiennes. Un tel travail repose sur une réflexion préalable sur les spécificités et les fonctions de cette épigraphie et sur l'observation de la façon dont numismates, linguistes et épigraphistes ont pu l'approcher.

\section{L'épigraphie monétaire du haut Moyen Âge}

\section{L'épigraphie utilitaire: lecture et identification}

L'étude des inscriptions monétaires est l'une des bases de la numismatique, en particulier pour les périodes mérovingienne et carolingienne. Ces légendes monétaires ont dès le XIX ${ }^{e}$ siècle donné lieu à des études lexicographiques et paléographiques, mais les savants s'y sont prioritairement intéressés de façon utilitaire, pour faciliter la lecture et les identifications. Aussitôt ces monnayages reconnus comme objets d'étude spécifiques, l'identification des lieux et des

1. L'université de Princeton a développé un tel outil pour le catalogue des sa collection numismatique (http://www.princeton.edu/ rbsc/department/numismatics/).

2. Unicode est une norme informatique, développée par le Consortium Unicode, qui vise à permettre le codage de texte écrit en donnant à tout caractère de n'importe quel système d'écriture un nom et un identifiant numérique, et ce de manière unifiée, quelle que soit la plate-forme informatique ou le logiciel. 
personnes dont le nom est inscrit dans les légendes a focalisé l'attention des numismates. Malgré des tentatives chronologiques comme celle de G. Depeyrot ${ }^{3}$, qui demeurent discutées, ce sont ces identifications qui ont servi de base aux classements des grands catalogues, et ce jusqu'à nos jours. Pour A. de Belfort, le classement géographique est le «seul rationnel ${ }^{4} »$, et c'est encore actuellement une position tout à fait défendable, malgré les incertitudes qui entourent le nom et la localisation de certains ateliers.

Ces identifications ont été et sont toujours l'occasion de nombreux débats. Non seulement il est loin d'être toujours possible d'établir une concordance entre le lieu de frappe exprimé et une localité moderne, mais la difficulté de lecture des légendes combinée à l'absence de normes de transcription fixes complique souvent les choses. De surcroît, la taille parfois inadaptée du flan monétaire, un mauvais alignement des coins avec celui-ci, ou une frappe inégalement répartie, peuvent nuire à la lisibilité de la légende. Le cas est particulièrement vrai pour les deniers mérovingiens, dont une proportion significative présente des flans carrés aux angles coupés et de taille réduite par rapport à celle des coins. Une meilleure connaissance de la paléographie et de la linguistique de la période, ainsi que des spécificités des inscriptions monétaires dans ces domaines, est donc toujours un élément crucial de l'amélioration de nos connaissances sur le monnayage franc altomédiéval.

C'est là l'opinion soutenue encore récemment par le linguiste Jean-Pierre Chambon ${ }^{5}$ dans un article de 2001. Il cherche à y dégager une méthode d'identification des ateliers monétaires mérovingiens qui soit conforme aux règles de la linguistique historique, mais aussi adaptée aux spécificités du corpus monétaire en question. Il remarque en effet que l'identification des ateliers monétaires fait rarement l'objet dans la littérature numismatique d'une critique argumentée. Elle repose bien plus souvent sur des évidences apparentes, et elle est rarement remise en cause une fois établie. Le préliminaire indispensable à cette méthode philologique est l'établissement assuré du texte, ce qui est loin d'être évident vu les difficultés de lectures que présentent les monnaies mérovingiennes.

\section{Épigraphie monétaire, épigraphie des inscriptions}

La linguistique et la paléographie des inscriptions mérovingiennes et carolingiennes ont fait l'objet d'études, certaines assez récentes, mais qui ne s'intéressent que peu aux inscriptions monétaires, lorsqu'elles les envisagent même. Les monnaies ont beau relever d'une science spécifique, les inscriptions qu'elles

3. G. Depeyrot, Le numéraire mérovingien, 4 vol., Wetteren, 1998; G. DePeyrot, Le numéraire carolingien. Corpus des monnaies, $3^{\mathrm{e}}$ édition augmentée, Wetteren, Paris, 2008.

4. A. de BELFORT, Description générale des monnaies mérovingiennes par ordre alphabétique des ateliers, Paris, 1892-1895, vol. I, p. V.

5. J.-P. Снамвоn, L'identification des noms d'ateliers monétaires mérovingiens (Arvernie et entours): points de vue de linguiste, $R N$ 2001, p. 347-405. 
portent justifient pourtant de les étudier sous l'angle de l'épigraphie. La légende est destinée à compléter ou à expliquer le message transmis par le type. Elle imite le langage officiel des inscriptions honoraires, mais le manque de place fait que les abréviations et les ligatures y sont souvent poussées à l'extrême.

Dans son étude sur la paléographie des inscriptions lapidaires ${ }^{6}$, Paul Deschamps suggère une étude comparative de la paléographie des inscriptions lapidaires, monétaires, sigillaires, et même des inscriptions peintes ou présentes sur les mosaïques, dont les lettres ont d'après lui des formes similaires. Bon nombre des observations qu'il fait concernant la pierre pourraient donc s'appliquer à une étude de l'épigraphie monétaire de la même période. Lorsqu'on compare les formes de lettres qu'il répertorie dans les inscriptions lapidaires à celles que l'on trouve à la même époque sur les monnaies, les parallèles sont évidents.

La variété des formes de lettres et les difficultés de lecture et d'interprétation qui caractérisent la période mérovingienne laissent place à la fin du VIII siècle à une épigraphie plus normalisée. La réforme de Charlemagne de l'hiver 793/794 marque un changement notable de ce point de vue: l'évolution est parallèle à celle des lettres formant les inscriptions lapidaires de l'époque, caractérisée par un retour à la capitale romaine à partir des dernières années du VIII ${ }^{\mathrm{e}}$ siècle $^{7}$. Des formes spécifiques demeurent toutefois et des particularités locales persistent au-delà de cette date, sur les monnaies comme sur les métaux et les pierres gravées. La véritable rupture du point de vue de l'épigraphie monétaire, dont l'évolution est dès lors distincte de celle des inscriptions lapidaires, s'amorce au cours du dernier tiers du $\mathrm{IX}^{\mathrm{e}}$ siècle.

Quant aux études linguistiques, si elles ne dédaignent pas l'apport des inscriptions monétaires, elles n'en font pas non plus un usage proportionné à son intérêt. Jules Pirson ${ }^{8}$ et surtout Henri d'Arbois de Jubainville ${ }^{9}$ en ont fait

6. P. Deschamps, Paléographie des inscriptions de la fin de l'époque mérovingienne aux dernières années du XII ${ }^{\mathrm{e}}$ siècle, Bulletin Monumental 1929, p. 5-81, p. 10-19 sur les périodes mérovingienne et carolingienne.

7. L'exemple le plus connu et le plus caractéristique de ce «renouveau» est l'épitaphe du pape Hadrien ${ }^{\text {er }}$ composée par Alcuin. J.-B. DE Rossi, L'inscription du tombeau d'Hadrien I ${ }^{\text {er }}$ composée et gravée en France par ordre de Charlemagne, dans Mélanges d'archéologie et d'histoire de l'École française de Rome, 8, 1888, p. 478-501.

8. J. PIRSON, La langue des inscriptions latines de la Gaule, Bruxelles, 1901.

9. H. D’Arbois de Jubainville, Etude sur la déclinaison des noms propres dans la langue franque à l'époque mérovingienne, Bibliothèque de l'École des chartes, 31, 1870, p. 312-352; H. D'Arbois DE JubainVILle, Études sur la langue des Francs à l'époque mérovingienne, Paris, 1900; M. Prou, Notes sur le latin des monnaies mérovingiennes, dans Mélanges de philologie romane et d'histoire littéraire offerts à Maurice Wilmotte à l'occasion de son $25^{e}$ anniversaire d'enseignement accompagné de fac-similés et d'un portrait, Genève, 1909, p. 522-540. On peut également signaler: P. BREILLAT, La langue des inscriptions monétaires mérovingiennes, Thèse pour l'obtention du diplôme d'archiviste paléographe, École nationale des chartes, 1935, et plus récemment, concernant le latin mérovingien en général, R. VERDO, La reconfiguration du latin mérovingien sous les Carolingiens. Étude socio-linguistique des diplômes royaux et des réécritures hagiographiques (VII - -IXe siècle), Thèse pour l'obtention du diplôme d'archiviste paléographe, École nationale des chartes, 2009. 
un usage modeste. Ils en tirent des éléments de réflexion sur l'orthographe, la phonétique, l'anthroponymie et la toponymie. Si la prosodie, la syntaxe et une bonne partie des champs traditionnels d'investigation de l'épigraphie et de la linguistique n'ont rien à y puiser, J.-P. Chambon note en revanche l'intérêt crucial du corpus numismatique mérovingien pour l'étude, à cette période, singulièrement mal éclairée sur le plan documentaire, de la transition du latin aux langues romanes.

Rares sont par ailleurs ceux qui ont fait de la paléographie des inscriptions monétaires un sujet d'étude à part entière. Jean Lafaurie a abordé le sujet en plusieurs articles portant sur des monnaies médiévales diverses, y compris mérovingiennes et carolingiennes, sans jamais cependant entreprendre d'étude synthétique.

Dans le domaine de l'épigraphie monétaire mérovingienne, l'étude d'une monnaie inédite de Bâle a été entre autres pour lui l'occasion de remarques ponctuelles ${ }^{10}$. Plus particulièrement, il suggère dans cet article de lier l'apparition dans les légendes de la forme delta du $\mathrm{D}$ et du $\mathrm{Z}$ aux tentatives de Chilpéric $\mathrm{I}^{\mathrm{er}}$ d'introduire dans l'alphabet latin de nouvelles lettres choisies dans l'alphabet grec. Il remarque notamment que ces formes sont particulièrement fréquentes en Neustrie, siège du pouvoir de ce roi.

Il a surtout fait ressortir l'incidence des techniques de gravure des coins sur la forme des lettres ${ }^{11}$. Un tournant majeur semble s'amorcer dès le milieu du $\mathrm{IX}^{\mathrm{e}}$ siècle, qui renouvelle les enjeux de l'épigraphie monétaire. Avec l'apparition de poinçons triangulaires pour graver les légendes, la variété stylistique des lettres, difficulté majeure pour les périodes antérieures, se trouve singulièrement réduite. Il s'agit en outre d'une évolution technique propre à la gravure des coins monétaires qui marque une divergence avec les inscriptions lapidaires.

\section{Paléographie et linguistique des monnaies du haut Moyen Âge}

Pour le haut Moyen Âge, la question de la paléographie des inscriptions n'a été traitée que de façon transversale dans les grandes études de la fin du XIX ${ }^{\mathrm{e}}$ et du début du $x^{\mathrm{e}}$ siècle: ainsi Joachim Lelewel ${ }^{12}$, Guillaume Conbrouse ${ }^{13}$, Arthur Engel et Raymond Serrure, Maurice Prou et Adrien Blanchet ${ }^{14}$ ont dressé des tableaux contenant les principales formes de lettres rencontrées. Il s'agit le plus souvent de remarques destinées à faciliter la lecture, données en introduction d'études plus générales de ces monnayages.

10. J. Lafaurie, Tremissis mérovingien inédit émis à Bâle (Un monnayage de Gontran en Burgondie), RN 1994, p. 182-198.

11. J. LAFAURIE, Epigraphie monétaire aux $\mathrm{X}^{\mathrm{e}}$ et $\mathrm{XI}^{\mathrm{e}}$ siècles, dans Actes du Congrès International de Numismatique. Paris, 6-11 juillet 1953, Commission internationale de numismatique, Paris, 1957 , p. 545-550.

12. J. LelEwel, Numismatique du Moyen Âge considérée sous le Rapport du Type, Paris, 1835.

13. G. Conbrouse, Description des monnaies royales de France, Paris, 1838.

14. Tome I de A. Blanchet et A. Dieudonné, Manuel de numismatique française, Paris, 1912-1936. 
G. Conbrouse ${ }^{15}$ est le plus complet sur la question de la paléographie, puisqu'il rassemble ses observations en deux planches d'alphabets exhaustifs, l'un pour les monnaies royales mérovingiennes, disposé de façon chronologique (figure 1), l'autre pour les monnaies de monétaires (figure 2). Il note une évolution chronologique de l'épigraphie, les lettres romaines d'origines se transformant souvent dans le cas des C, D et O en lettres carrées, et en onciale dans le cas du E.

Pour A. Blanchet ${ }^{16}$, les formes de lettres sont si nombreuses qu'il est inutile de toutes les signaler; il en retient seulement quelques-unes. Il privilégie une interprétation liée à l'époque plutôt qu'à des régions déterminées pour les variations de la forme des lettres, mais il considère qu'il y en a au moins autant qui sont dues à la technique maladroite des graveurs, constituant une paléographie accidentelle et donc secondaire. Il laisse en outre de côté toute considération sur les liaisons de lettres.

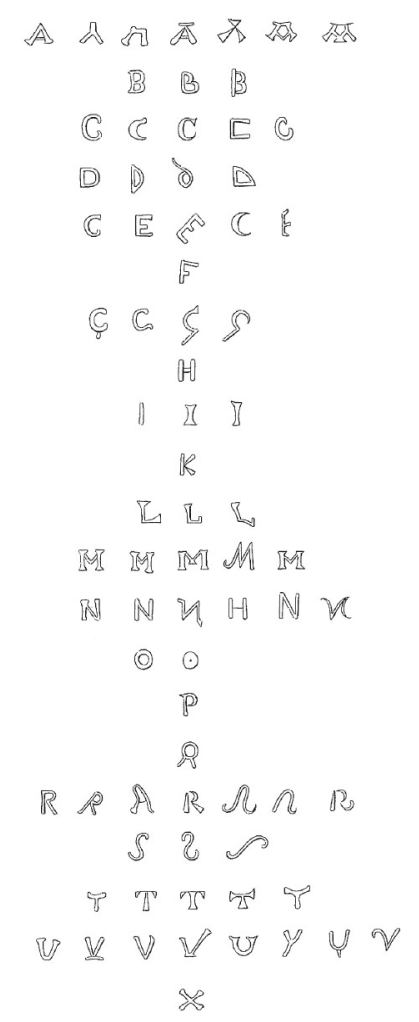

Figure 1 - «Alphabet royal mérovingien» (planche II de G. Conbrouse 1838).

15. G. Conbrouse, op. cit. n. 13.

16. A. Blanchet et A. Dieudonné, op. cit. n. 14. 


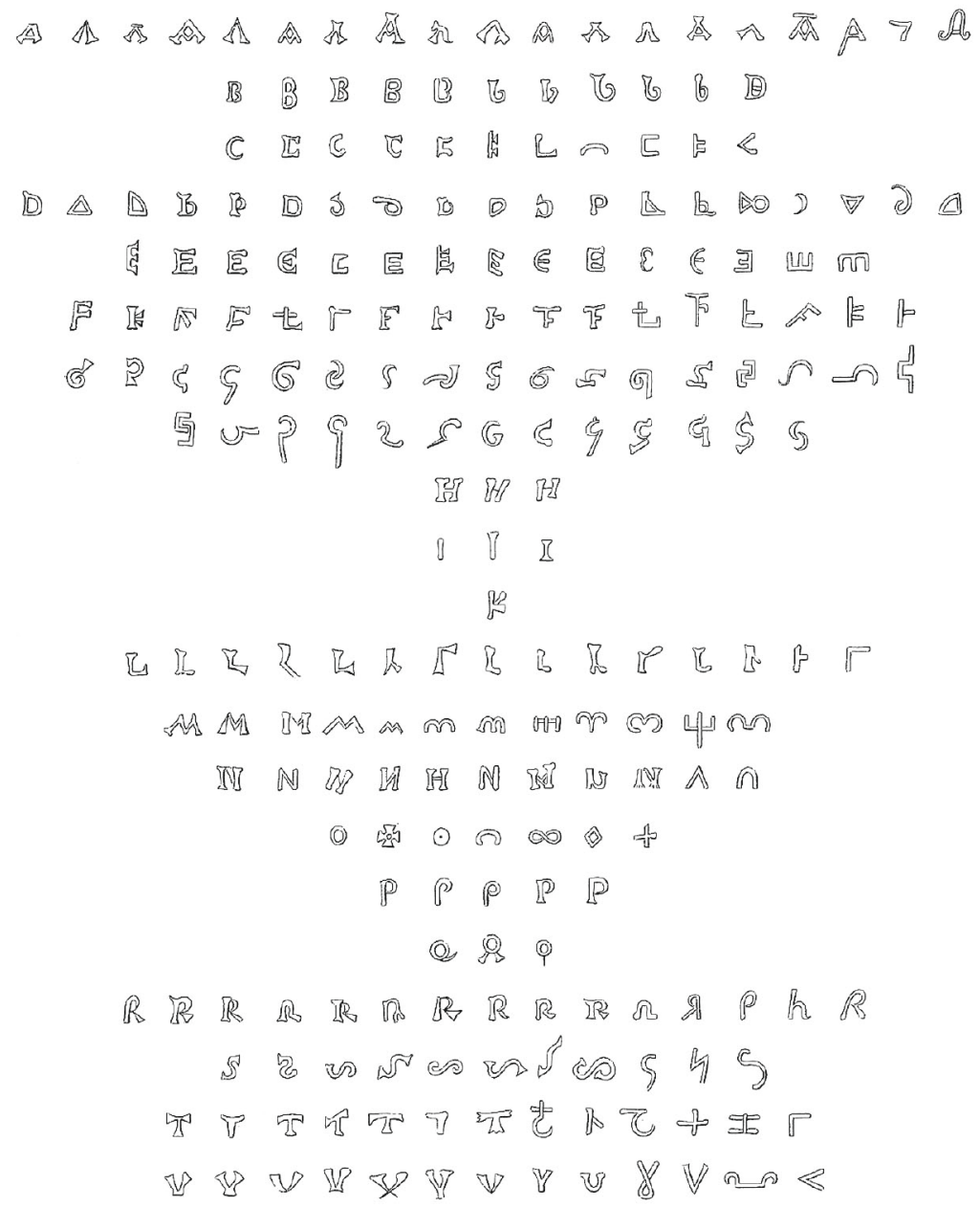

Figure 2 - «Alphabet des monétaires» (planche III de G. Conbrouse 1838).

Ce dernier point avait été abordé par J. Lelewe ${ }^{17}$. Outre des remarques sur l'apparition à la fin de l'époque mérovingienne des formes carrées du C, du D et du $\mathrm{O}$, en particulier sur le monnayage d'argent, il avance une explication d'ordre graphique et esthétique à certaines particularités des légendes monétaires de cette période. Deux éléments sont d'après lui à prendre en compte : le remplissage de la totalité du pourtour de la monnaie par la légende, et la

17. J. LELEWEL, op. cit. n. 12, p. 30. 
recherche de la symétrie. Il interprète ainsi la plupart des ligatures comme une conséquence directe de la place réduite disponible sur la monnaie. La présence de certains $\mathrm{S}$ couchés s'explique d'après lui par une tentative de combler un vide, et certaines lettres ou jambages comme des éléments graphiques sans signification y sont placés pour équilibrer la légende. Le choix de certaines formes de lettres ou de graphies peut également dépendre d'une recherche de symétrie.

Ces considérations, quoique souvent invalidées par des réinterprétations et des relectures plus récentes, restent utiles, en particulier en regard des légendes factices que l'on trouve sur certaines monnaies (figure 3). Il s'agit de légendes purement décoratives, en général composées essentiellement de jambages, et dépourvues de toute signification, que l'on trouve sur une partie du monnayage mérovingien. Elles semblent accréditer l'idée que certains graveurs de coins étaient illettrés, et qu'ils copiaient les légendes monétaires sur un mode purement graphique. Les variations dans la forme des lettres et dans leur orientation, ainsi que certaines erreurs et modifications, seraient alors parfaitement explicables bien qu'indéfendables sur le plan orthographique ou linguistique.
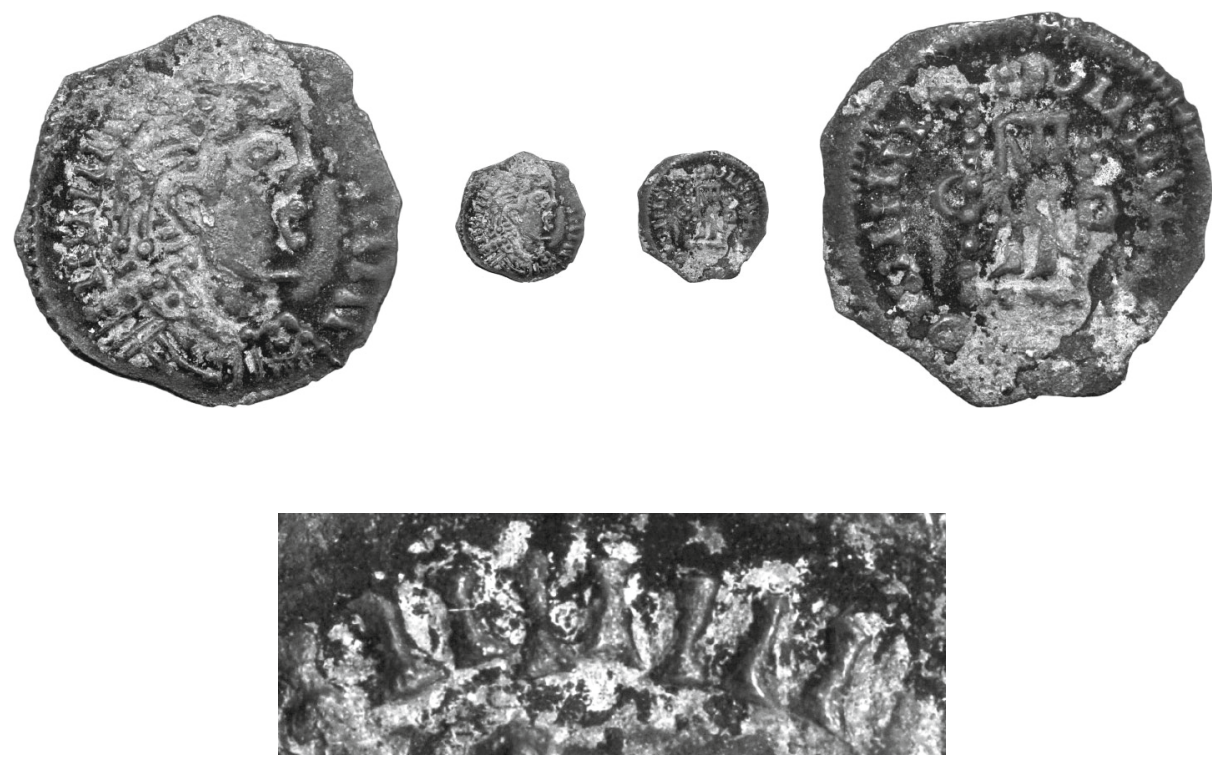

Figure 3 - Exemple de monnaie mérovingienne avec une légende factice composée de traits verticaux (BnF Prou, Mérov., 28). Haut: échelle 1:1 au centre et agrandissement 3:1 autour; bas : agrandissement de la légende verticale à gauche du buste au droit. 
Cécile Treffort présente des idées similaires à l'occasion d'un article sur une série de boucles de ceinture des $\mathrm{V}^{\mathrm{e}}-\mathrm{VI}^{\mathrm{e}}$ siècles découvertes en Burgondie, essentiellement en contexte funéraire ${ }^{18}$. Elles portent des inscriptions souvent difficilement lisibles, que l'auteur interprète comme ayant une fonction prophylactique. Ces objets la conduisent à soulever la question des inscriptions «pseudo-épigraphiques» et de leur signification. Souvent interprétées comme la forme dégénérée d'un texte antérieur, elles pourraient correspondre plutôt à un mode de remplissage de l'espace par des lettres choisies pour des raisons esthétiques, ou même dans certains cas magiques. Sans aller jusque là pour les légendes monétaires, il faut admettre que l'explication esthétique est dans certains cas défendable.

L'étude la plus aboutie des inscriptions monétaires pour le haut Moyen Âge revient à $\mathrm{M}$. Prou et ses propres recherches tiennent en quelques dizaines de pages. En 1909, il consacre vingt pages au latin des inscriptions monétaires ${ }^{19}$. Il y souligne la pertinence de l'utilisation des inscriptions monétaires pour l'étude des évolutions phonétiques, car elles se composent essentiellement de noms propres, qui sont particulièrement sensibles à ces évolutions. Les phénomènes qu'il met en avant n'ont rien de très original: épenthèse du G, chute de la dentale, résistance ou non de l'S final au nominatif des noms de la deuxième déclinaison, évolution du verbe fieri. Plus intéressantes dans la perspective paléographique et épigraphique qui est la nôtre sont les difficultés qu'il souligne dans l'utilisation des légendes de monnaies par le linguiste. D'une part, la forme des lettres et les techniques de gravure peuvent rendre incertaine la lecture des légendes. La négligence ou l'illettrisme des graveurs peut d'autre part conduire à l'oubli de certaines lettres ou à leur déformation sans pour autant que ces changements aient une quelconque signification sur le plan de la langue. Au fil des imitations de types, les légendes peuvent devenir de simples ornements graphiques, sans aucune signification.

Quant à l'étude paléographique des inscriptions, M. Prou en traite dans son catalogue des monnaies mérovingiennes de la Bibliothèque nationale, en à peine trois pages d'appendice ${ }^{20}$. Il se contente de signaler les formes possibles des différentes lettres pour faciliter la lecture des légendes, sans en tirer de conséquences paléographiques ou numismatiques plus importantes. D'après lui, les modifications de la forme des lettres sont trop infimes pour fournir un argument chronologique ou typologique exploitable.

18. C. TREFFORT, Vertus prophylactiques et sens eschatologique d'un dépôt funéraire du haut Moyen Âge: les plaques boucles rectangulaires burgondes à inscription, Archéologie médiévale, XXXII, 2002, p. 31-53.

19. M. Prou, op. cit. n. 9., p. 522-540.

20. Prou, Mérov., p. CXVI-CXVIII. 
Tous ces savants mettent en avant la nécessaire prudence méthodologique à adopter dans l'étude de ces inscriptions monétaires. Si beaucoup de phénomènes constatés sont attribués à l'illettrisme éventuel des graveurs, à leur maladresse, ou à des impératifs esthétiques (remplissage de la légende, composition du type), il reste que les légendes attestent de phénomènes linguistiques significatifs, et qu'une interrogation sur leur forme, y compris du point de vue paléographique, reste légitime.

\section{Le rendu des légendes dans les publications}

\section{Les caractères métalliques}

Pour transcrire les légendes monétaires, la solution choisie par les traités et par les grands catalogues de monnaies mérovingiennes du $\mathrm{XIX}^{\mathrm{e}}$ siècle et du début $\mathrm{du} \mathrm{xx}^{\mathrm{e}}$ siècle, en particulier le catalogue de M. Prou et celui de A. de Belfort, a été de constituer un jeu de caractères d'imprimerie spécifique destiné à rendre quelques grandes variations possibles. Malgré les possibilités théoriquement infinies offertes par les plombs, il s'agit le plus souvent d'une sélection. L'éditeur de l'ouvrage de J. Lelewel ${ }^{21}$ fait remarquer «la nature de l'impression de cet ouvrage, bien différente de la majeure partie des livres destinés au public. Les recherches et les observations sur les monnaies expliquées ou mentionnées dans le texte ont dû être accompagnées de caractères du moyen-âge, d'emblèmes, de symboles ; plusieurs monnaies ont été insérées dans le corps même des pages imprimées en caractères ordinaires ; sans cela, le lecteur eût été renvoyé à chaque instant aux planches, et son attention, en se partageant, eût été affaiblie. Un coup d'œil, jeté sur les nombreuses pages remplies de caractères hétérogènes, suffira pour faire apprécier la vérité de ces observations».

Parmi les formes que M. Prou signale dans son étude paléographique, seules quelques-unes sont retenues ensuite dans la transcription des légendes de son catalogue. Certains détails qu'il note mais néglige d'inclure dans son jeu de plombs, par exemple les lettres dont la haste peut dépasser les membres transversaux, se retrouvent sous la même forme dans les inscriptions lapidaires signalées par Edmond Le Blant pour les $\mathrm{VI}^{\mathrm{e}}$ et $\mathrm{VII}^{\mathrm{e}}$ siècles $^{22}$. Il eût été intéressant de les prendre en compte afin d'étudier la chronologie des monnaies présentant les mêmes caractéristiques. M. Prou a cherché en priorité à distinguer les lettres de module différent, alphabet capital et alphabet oncial ; les plombs pouvaient en outre être retournés pour rendre les lettres inversées ou couchées.

Comment expliquer ce choix de ne pas inclure toutes les variantes possibles des lettres présentes dans les légendes monétaires dans son jeu de caractères

21. J. LeLEWEL, op. cit. n. 12, p. 9-10.

22. E. Le Blant, Nouveau recueil des inscriptions chrétiennes de la Gaule antérieures au VIII ${ }^{e}$ siècle, Paris, 1892. 
typographiques? On peut avancer d'une part des contraintes techniques et financières, et d'une autre des impératifs scientifiques.

Les lettres gravées sur les monnaies du haut Moyen Âge sont bien moins standardisées que pour d'autres monnayages. Un rendu exact nécessiterait presque de fondre un caractère typographique par lettre de légende, ce qui est matériellement impossible, et scientifiquement peu intéressant. Il faut en outre que la casse employée soit utilisable. La création et la production de caractères en métal sont depuis les origines des opérations longues, hautement techniques et coûteuses, ce qui limite fortement le nombre de caractères spéciaux envisageables. Ces caractères métalliques, une fois réalisés, sont de plus la propriété de l'imprimeur qui les a fait faire, d'où une utilisation restreinte. Il ne fait pas de doute que la constitution d'une telle casse n'était guère rentable d'un point de vue commercial, et c'est donc une initiative isolée que celle des catalogues de M. Prou.

Autre écueil, la part inévitable d'interprétation dans le rendu par l'imprimerie de la forme des caractères présents sur les monnaies. La transcription des légendes implique de réduire la diversité des formes à une gamme limitée et relativement unifiée de caractères, dans le but de donner une lecture aisément compréhensible. Si la légende est difficilement lisible ou l'interprétation peu sûre, cette transcription doit aussi dans l'idéal donner au lecteur des pistes de réflexion. Enfin, le degré d'exactitude retenu dans la légende doit coïncider avec les objectifs de la publication. Selon l'étude entreprise, une simple lecture de la légende peut suffire, et par conséquent un jeu de caractères normaux. Dans d'autres cas, on cherche à repérer et à mettre en série certaines caractéristiques graphiques, qui devront dans la mesure du possible figurer dans la transcription de la légende.

Une difficulté supplémentaire apparaît lorsqu'on passe de l'imprimerie traditionnelle à l'imprimerie assistée par ordinateur. L'absence de police de caractères correspondant à la casse du catalogue d'origine fait qu'il est impossible de recomposer l'ouvrage. C'est pourquoi toutes les rééditions des catalogues de M. Prou par exemple sont des fac simile des originaux, technique coûteuse et qui interdit toute correction ou modification.

\section{Caractères standard et illustration}

L'adjonction de planches d'illustration à des notices en caractères standard est souvent perçue comme la meilleure solution. Pour une étude des techniques de gravure des coins, par exemple, la précision requise rend impossible l'utilisation d'une police de caractères: c'est vers la photographie ou, par le passé, vers le dessin, la gravure ou l'empreinte, qu'il faut se tourner. Le problème est le même en ce qui concerne l'étude des liaisons de coins ou de l'évolution des types ${ }^{23}$.

23. D. Hollard, L'illustration numismatique au XIX ${ }^{\mathrm{e}}$ siècle, $R N$ 1991, p. 7-42. 
Le recours à des illustrations dispense l'auteur d'inclure dans son texte des caractères différents des caractères d'imprimerie standard, ou de sa police de travail de départ. Il évite aussi des ajustements parfois délicats avec l'imprimeur, qui peuvent peser lourd dans le prix de l'édition. Il est en effet aussi possible d'intégrer les légendes dans la notice en mode image, ou sous la forme de polices personnelles, mais dans les deux cas des problèmes de transmission et d'utilisation par les imprimeurs se posent. C'est pourquoi les éditeurs de publications numismatiques demandent encore aux auteurs d'éviter d'utiliser de telles polices, voire leur recommandent d'utiliser la police spécifique de la revue ${ }^{24}$.

Le choix d'une police standard n'est pas exempt de désagréments. Une interprétation des légendes très poussée s'impose, or la lecture de ces légendes est loin d'être aisée ou univoque (voir figure 4 pour un exemple particulièrement ardu). Elles sont parfois rognées, mal imprimées sur le flan, difficilement lisibles; certains caractères peuvent être retournés et se confondre avec d'autres; enfin elles peuvent même comme nous l'avons vu n'être que l'interprétation graphique d'une légende antérieure, ou même un simple élément décoratif, sans signification. Les légendes peuvent en outre être internes, externes, rétrogrades, ou même un savant mélange de tout cela, toutes choses qu'une police standard n'est absolument pas apte à rendre et qui ne se retrouve par conséquent que dans les planches, ou, dans le meilleur des cas, dans la description du type.
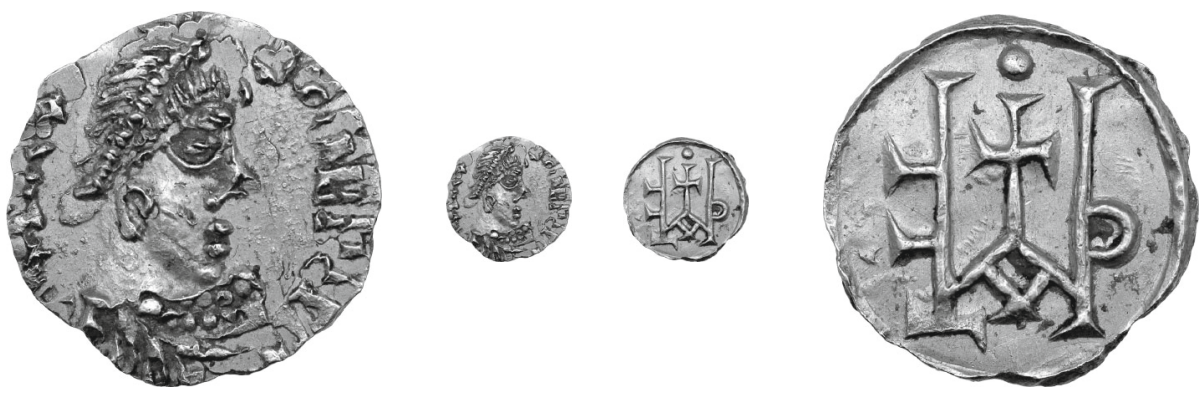

Figure 4 - Exemple de monnaie mérovingienne avec une légende presque illisible au droit, probablement surfrappée. On distingue un R rétrograde interne. Pour de telles légendes, la possibilité d'indiquer l'orientation de chaque lettre, ainsi que la présence de caractères non identifiables dans la transcription est essentielle. (Mérovingienne Prou 243). Échelle 1:1 au centre et agrandissement 3:1.

24. Voir à la fin de ce numéro de la Revue Numismatique, ou pour Numismatic Chronicle: http://www.numismatics.org.uk/articles/guidance-for-contributors-to-the-numismatic-chronicle. 
Sans en rester à une police standard, quelques adaptations peuvent dans certains cas suffire. C'est le cas de la police développée par Jacqueline PiletLemière $^{25}$, qui inclut seulement quelques caractères rétrogrades et quelques formes particulières.

\section{Des polices d'approche purement graphique}

Depuis vingt ans, le nombre de polices de caractères s'est multiplié, et en particulier celles de type paléographique ${ }^{26}$. C'est la transformation des conditions techniques qui a permis cet essor, d'abord le développement dans les années 1960 de la photocomposition, et surtout à partir de la fin des années 1970 celui de la typographie numérique. Le marché et les conditions économiques de la production en ont été bouleversés, et les prix ont chuté. La micro-informatique a parachevé cette évolution. Avec l'apparition de logiciels spécialisés et les possibilités de diffusion par internet, les années 1990 ont vu la multiplication des «micro fonderies », plus ou moins durables, souvent composées d'une seule personne et produisant des polices innombrables et peu onéreuses. La plupart des polices produites restent cependant dans une logique graphique et éditoriale.

Pour certaines études très spécifiques, il peut s'avérer intéressant de créer une police de toutes pièces. Cela permet un rendu précis des particularités graphiques intéressant l'étude, mais soulève des questions de méthodologie et de rentabilité. Le problème se pose toujours du degré de précision retenu dans le dessin des lettres, et des confusions et erreurs qui peuvent découler de ces choix. Étant très spécifique, la police n'a par ailleurs pas vocation à être largement utilisée et partagée. Sont présentés ici les exemples de quelques polices moins spécifiques, mais plus intéressantes pour la communauté numismatique, et pour le haut Moyen Âge en particulier ${ }^{27}$.

\section{Numismatica Medievalis}

La police Numismatica Medievalis a été lancée par J. Moens pendant son mandat de secrétaire de la Société Royale de Numismatique de Belgique. Ce sont avant tout les frais croissants de mise en page la Revue belge de numismatique qui ont motivé cette initiative; il s'agissait de transmettre à l'imprimeur un document prêt à l'impression, et comportant donc des polices utilisables pour le traitement de texte et pour l'impression. Il n'existait cependant pas de police,

25. J. Lafaurie et J. Pilet-Lemière, Monnaies du Haut Moyen Âge découvertes en France ( $V^{e}$-VIII ${ }^{e}$ siècle), (Cahiers Ernest Babelon 8), Paris, 2003.

26. M. Sмітн, Du manuscrit à la typographie numérique: présent et avenir des écritures anciennes, Gazette du livre médiéval, 2008, p. 51-78.

27. D'autres expériences ont été menées collectivement comme pour le Corpus numorum saeculorum IX-XI, qui ne recoupe qu'en partie cette période, mais nous nous en tenons à deux exemples. 
libre ou commerciale, qui satisfît réellement aux besoins des numismates en termes de rendu graphique des caractères présents sur les monnaies. Il fallait en créer une.

La première étape a consisté à scanner dans des ouvrages numismatiques du $\mathrm{XIX}^{\mathrm{e}}$ siècle - et donc libres de droits - des caractères ; dans un second temps, en 2010 et 2011, un appel à collaboration publié dans la Revue belge de numismatique a permis l'ajout de nouveaux caractères selon les besoins des utilisateurs. Numismatica Medievalis est donc toujours en cours de transformation.

Si Numismatica Medievalis est effectivement utilisée depuis 2011 pour la Revue belge de numismatique et avant cela pour le Bulletin du Cercle d'études numismatiques, elle ne dispense pas ces revues d'utiliser encore d'autres caractères sur mesure pour les besoins de certains articles. En sus des quelque deux mille caractères «officiels» de la police, des ajouts peuvent aisément être pratiqués par les utilisateurs selon leurs besoins à partir d'autres polices, d'images ou de dessins de caractères. Il va donc sans dire que ce n'est pas une police indexée dans unicode ${ }^{28}$.

L'autre problème principal de cette police est le très large champ chronologique qu'elle embrasse, «des premières émissions jusqu'à la Renaissance ${ }^{29}$ ». Elle est donc pour l'instant assez hétérogène, et ne procède pas de choix raisonnés sur un corpus déterminé, mais plutôt d'une accumulation en fonction des besoins. L'idée a été avancée de la subdiviser en plusieurs versions plus spécifiques en fonction des époques et des monnayages, pour rendre l'entreprise plus cohérente et utilisable.

\section{Inscription Numismatic}

Comme la précédente, cette police a vu le jour pour les besoins de l'édition numismatique, lorsque les plombs existants sont devenus obsolètes. Inscription Numismatic est actuellement la propriété de la British Academy. Elle a été créée par Michael Metcalf à partir de la police des presses universitaires d'Oxford (Oxford University Press), qui venaient juste de cesser leurs activités d'imprimeurs. Elle a été utilisée pour la première fois pour les volumes des Sylloge of Coins of the British Isles ${ }^{30}$.

Elle répond cependant à une logique toute différente en ce qui concerne sa composition et son mode d'accroissement. Inscription Numismatic se veut une police stylisée; elle n'a pas pour ambition de reproduire exactement la forme des lettres d'origine (voir la Table des caractères présentée en figure 5). L'ajout de nouveaux caractères est très contrôlé, et passe exclusivement par les éditeurs de Numismatic Chronicle, et plus précisément par R. Hodges. En dépit

28. Sur unicode, voir supra p. 262 (note 2).

29. http://www.numisbel.be/NumisMed_FR.htm.

30. Description du projet: http://www.fitzmuseum.cam.ac.uk/coins/projects/scbi/index.html. 


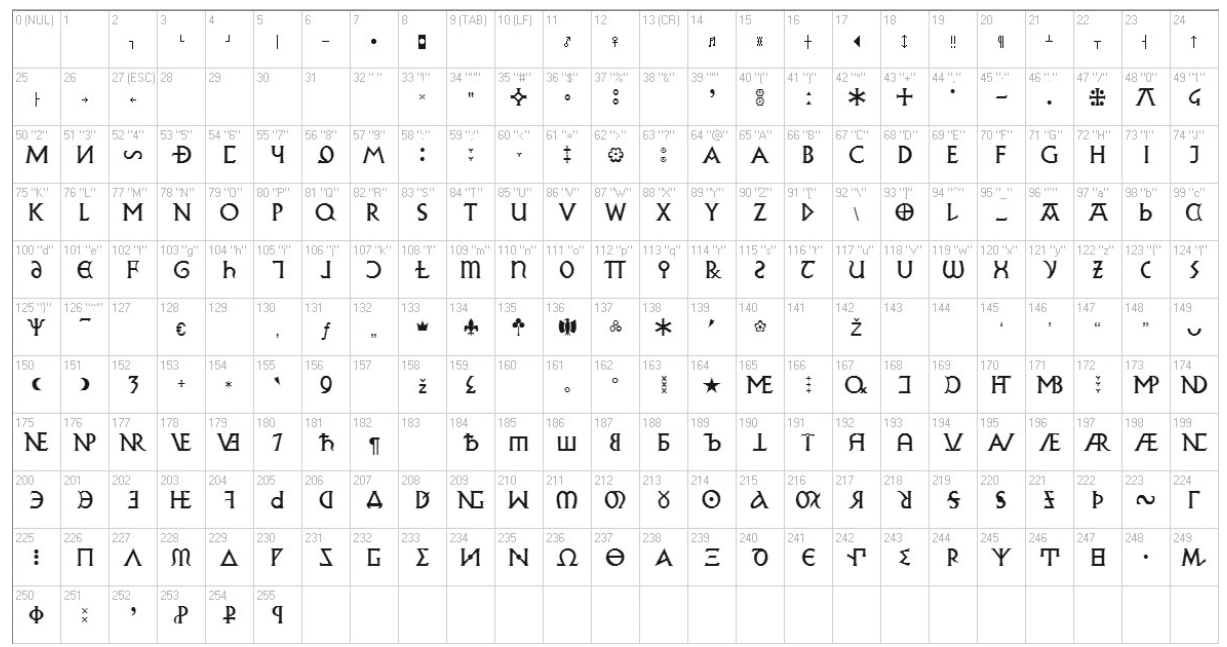

Figure 5 - Table des caractères de la police Inscription Numismatic.

du peu de liberté d'action des utilisateurs, qui ne sont pas libres d'ajouter leurs propres caractères, une certaine flexibilité existe. Pour la publication du trésor de Pimprez ${ }^{31}$ notamment, une version particulière a été développée par R. Hodges.

\section{De nouvelles perspectives pour les polices numismatiques}

\section{Des possibilités techniques et des usages renouvelés}

Outre les besoins de l'édition papier que nous avons déjà soulignés, la pertinence d'une réflexion sur les polices numismatiques est accentuée par le fait que de plus en plus d'institutions mettent leurs collections en ligne, avec une quantité d'information et des possibilités de recherche variables. L'université de Princeton par exemple permet une recherche par monogramme, lettres de forme particulière, numéros et caractères non traditionnels chinois ${ }^{32}$. Dans l'ensemble, les solutions implémentées par les diverses institutions dépendent de leurs besoins et possibilités techniques.

Les formats techniques ont connu en trente ans une évolution rapide sur laquelle il est inutile de s'attarder en détail mais qui ouvre des possibilités sans précédent, surtout grâce au format de police Open Type et à Unicode. Open Type offre plusieurs avantages pour la reproduction des écritures anciennes : la

31. The Pimprez hoard and other important proprierties [Vente Spink and son, Londres, 06 et 07/10/2004], Londres, 2004.

32. http://www.princeton.edu/ rbsc/department/numismatics/database\% 20 search.html. 
possibilité d'avoir un grand nombre de caractères, et des fonctionnalités comme la gestion des ligatures.

Quant à Unicode, le terme désigne à la fois le consortium crée en 1989 par divers acteurs du monde informatique (entreprises, institutions, chercheurs, éditeurs...), et le jeu de caractères qu'il développe et promeut. Un jeu de caractères consiste en un répertoire de codages et les caractères correspondants. Pour chaque caractère, le répertoire inclut si possible un glyphe, c'est-à-dire la manifestation graphique du caractère, un nom, et des caractéristiques d'utilisation. Le jeu de caractères Unicode repose sur des conventions normalisées ; un des objectifs principaux du consortium est d'unifier dans la mesure du possible les normes antérieures. Cela offre l'avantage de garantir la transmissibilité des polices de caractères compatibles Unicode.

C'est un jeu de caractères ouvert, c'est-à-dire qu'en plus d'inclure des caractères relevant de divers types d'écriture et des caractères de commande et techniques, il comporte des codages à usage privé et des plages réservées pour des évolutions futures. Les médiévistes y ont déjà fait inclure des caractères particuliers, et coordonnent leurs initiatives grâce au Medieval Unicode Font Initiative (MUFI) ${ }^{33}$.

Ces possibilités techniques nouvelles ne sont cependant pas toujours faciles à exploiter. Leur mise en œuvre demande un certain savoir-faire. Quelques applications permettent d'en tirer pleinement parti, mais ce sont souvent des logiciels complexes, professionnels ou coûteux. Ces applications ne dispensent pas d'une réflexion préalable: toute imitation d'écriture ancienne doit préciser d'emblée ses finalités pour justifier ses choix, entre fidélité et l'adaptation à des usages pratiques. Selon les cas, l'option la plus pertinente peut être plus ou moins complexe et élaborée.

\section{Athena Ruby: un modèle}

Le Dumbarton Oaks Center for Byzantine Studies a annoncé pour 2012 une nouvelle police de caractères, Athena Ruby, destinée à transcrire les inscriptions monétaires et sigillographiques pour le domaine byzantin ${ }^{34}$. De fait, il s'agit de la refonte de plusieurs polices développées par cette institution depuis le début des années 1980 pour les études byzantines.

La première de ces polices, qui prit par la suite le nom d'Athena, était une police True Type développée sur Macintosh par Nicolas Oikonomides pour les publications de sigillographie byzantine de Dumbarton Oaks. C'était une véritable révolution: Athena permettait à la fois de transcrire les ligatures, les abréviations, les variantes de forme des lettres et de produire sur un ordinateur personnel un

33. http://www.mufi.info.

34. De plus amples informations sur cette police et sur l'avancement du projet sont disponibles sur le site de Dumbarton Oaks à l'adresse suivante: http://www.doaks.org/resources/publications/ athena-ruby-inscription-font. Une version béta peut d'ores et déjà être demandée aux concepteurs. 
document prêt à imprimer, et ce dès le milieu des années 1980. Dans les années 1990, le Département des Publications, sous l'impulsion notamment de son directeur Gl. Ruby, créa plusieurs nouvelles polices, cette fois destinées spécialement à l'épigraphie monétaire: Grierson, Grierson Variants, Coin Art, Coin Greek, et Coin Inscription. À la mort de Gl. Ruby, un projet d'unification des polices existantes partiellement financé par un petit legs à sa mémoire fut adopté, sous la maîtrise d'œuvre de J. Kalvesmaki.

Athena Ruby est sans doute le projet de police de caractères numismatiques le plus intéressant et le plus innovant à ce jour. C'est une police Open Type libre, compatible Unicode et agréée par ce consortium, permettant de transcrire une vaste gamme de lettres, signes et combinaisons de lettres choisis de façon cohérente et raisonnée en fonction d'un domaine de recherche spécifique, avec un réel souci de communicabilité de compatibilité. Elle est en revanche limitée dans son usage aux études byzantines, bien qu'elle propose un nombre intéressant de caractères utilisables pour la numismatique de l'Occident altomédiéval.

\section{Conclusion}

Les perspectives offertes par les polices de caractères numériques sont stimulantes et pourraient, dans un avenir proche, redynamiser les études d'épigraphie monétaire des époques mérovingienne et carolingienne. La réflexion préalable qu'elles appellent en termes de standardisation des caractères, de formalisation et de normalisation dans le rendu des légendes permettent d'envisager des projets collectifs à plus grande échelle sur les monnaies mérovingiennes et carolingiennes. La constitution de bases de données homogènes notamment offre la possibilité d'enquêtes nouvelles, sur des corpus élargis.

La création d'une police à la fois pertinente scientifiquement et suffisamment simple d'utilisation pour en généraliser l'emploi pourrait en effet faciliter les recherches de caractéristiques régionales ou chronologiques, ainsi que la lecture de certaines légendes problématiques. Elle serait le complément nécessaire à l'émergence de bases de données en ligne en proposant un outil commun et une normalisation des usages de transcription entre numismates. Elle pourrait être également l'occasion d'une ouverture vers les autres supports épigraphiques de l'époque, jusqu'alors étudiés séparément. La prise en compte de toutes les fonctionnalités souhaitables pour une étude optimale des inscriptions monétaires du haut Moyen Âge conduit cependant à sortir du strict cadre des possibilités techniques offertes par la police de caractères. Peut-être faudrait-il imaginer un outil nouveau du point de vue épigraphique comme informatique ${ }^{35}$.

35. Les auteurs remercient $\mathrm{M}^{\mathrm{me}}$ Cécile Morrisson ainsi que MM. Jan Moens et Marcus Phillips pour les informations qu'ils ont bien voulu leur communiquer sur les polices Athena Ruby, Numismatica Medievalis et Inscription Numismatic, respectivement. Ils sont également reconnaissants M. Marc Bompaire pour ses conseils et suggestions avisés. 
